\title{
The efficacy of mitochondrial targeting antiresistant epirubicin liposomes in treating resistant leukemia in animals
}

This article was published in the following Dove Press journal:

International Journal of Nanomedicine

| December 201 |

Number of times this article has been viewed

\author{
Ying Men* \\ Xiao-Xing Wang* \\ Ruo-Jing Li \\ Yan Zhang \\ Wei Tian \\ Hong-Juan Yao \\ Rui-Jun Ju \\ Xue Ying \\ Jia Zhou \\ Nan Li \\ Liang Zhang \\ Yang Yu \\ Wan-Liang Lu
}

State Key Laboratory of Natural and Biomimetic Drugs, and School of Pharmaceutical Sciences, Peking University, Beijing, People's Republic of China

*These authors contributed equally to this manuscript
Background: Multidrug resistance (MDR) of cancers can be circumvented by inducing programmed cell death, which is known as apoptosis. Mitochondria play a crucial role in apoptosis. Mitochondria-specific therapy would provide an efficient strategy for treating resistant cancers.

Design and methods: A strategy was proposed here to overcome MDR by designing cancer mitochondria-specific drug-loaded liposomes, namely, antiresistant epirubicin mitosomes, aimed at treating resistant leukemia by targeting mitochondria. Evaluations were performed on human chronic leukemia K562, MDR K562/ADR cells, and female BALB/c nude mice xenografted with MDR K562/ADR cells. The liposomes were characterized through assays of cytotoxicity, mitochondrial targeting, caspase-9 and caspase-3, antitumor activities, and TUNEL (terminal deoxynucleotidyl transferase dUTP nick end labeling) analysis.

Results: The average size of antiresistant epirubicin mitosomes was in the range of 105-115 nm. Antiresistant epirubicin mitosomes were effective in inhibiting proliferation of MDR K562/ADR cells in vitro and selectively accumulated into the mitochondria. Caspase- 9 and caspase- 3 activity was increased after applying antiresistant epirubicin mitosomes. In xenografted resistant MDR K562/ADR tumor in nude mice, antiresistant tumor effect of antiresistant epirubicin mitosomes was evidently observed. Apoptotic inducing effects by antiresistant epirubicin mitosomes were noticeably evidenced via mitochondrial pathway.

Conclusions: Antiresistant epirubicin mitosomes had significant inhibitory effect against resistant leukemia in vitro and in vivo, hence providing a promising strategy for improving therapeutic efficacy in resistant human leukemia.

Keywords: mitosomes, mitochondria signaling pathway, nude mice

\section{Introduction}

Multidrug resistance (MDR) is a major obstacle to successful cancer chemotherapy, and has been evidenced in a variety of cancers, including leukemia and breast, ovarian, lung, and lower gastrointestinal tract cancers. ${ }^{1}$ The major mechanisms of MDR could be derived from acquired and/or intrinsic factors. ${ }^{2}$ Acquired resistance develops during chemotherapy, resulting in the following outcomes: (1) less drug accumulation in cancer cells due to the efflux of drugs by overexpression of adenosine triphosphate (ATP)-binding cassette (ABC) transporters; (2) an enhanced drug-detoxifying function of cancer cells due to the enhanced deoxyribonucleic acid (DNA) repair ability and the increased activity of cytochrome P-450; and (3) decreased sensitivity of apoptosis due to the alteration of caspases. ${ }^{3}$ In contrast, intrinsic resistance already exists at the beginning of chemotherapy. It may be derived from (1) host factors,
Correspondence: Wan-Liang Lu Department of Pharmaceutics, School of Pharmaceutical Sciences, Peking University, Xueyuan Road 38, Beijing 100191, People's Republic of China

Tel +861082802683

Fax +86 I0 82802683

Email luwl@bjmu.edu.cn 
(2) the genetic/epigenetic alternations such as activation of oncogenes and inactivation of tumor suppressor genes, or (3) the alternation of the apoptosis pathways. ${ }^{4}$ Most likely, the MDR of leukemia is due to the intrinsic resistance.

Mitochondria are the powerhouses of cells, and the process of creating cell energy is known as cellular respiration. Most chemical reactions involved in cellular respiration happen in the mitochondria. The process by which mitochondria synthesize ATP by oxidative phosphorylation via the respiratory chain creates a transmembrane electrochemical gradient. ${ }^{5}$ The membrane potential of mitochondria in vitro is estimated to be in the range of $180-200 \mathrm{mV}, 6$ while the membrane potential of mitochondria in living cells and organisms is lower $(130-150 \mathrm{mV})$ due to metabolic processes such as ATP synthesis and ion transport. ${ }^{7}$ This feature makes it possible to accumulate organic cations into mitochondria. Sufficient lipophilicity and delocalization of the positive charge are the prerequisites for mitochondrial accumulation of organic cations in response to the mitochondrial membrane potential. ${ }^{8}$

Mitochondria are also known to play a role in programmed cell death via apoptosis. ${ }^{9}$ Generally speaking, there are two semi-interdependent routes leading to apoptosis. ${ }^{10}$ One route involves the ligand binding to death receptors at the cell surface, ${ }^{11}$ while another involves mitochondria. ${ }^{12}$ In the latter route, apoptotic inducer induces an increase in the permeability of the outer membrane, causing the release of cytochrome $c,{ }^{13}$ and therefore triggering apoptosis. Cytochrome c may be released from mitochondria to the cytosol through Bax/Bak channels, permeability transition (PT) pores, or damaged mitochondrial membrane. ${ }^{13}$ Meanwhile, the activated caspase family leads to a cascade of events in the cytosol, ${ }^{14}$ eventually resulting in partial self-digestion of cells. There are two kinds of apoptotic gene-encoded proteins on the outer membrane of the mitochondria. One is pro-apoptotic gene-encoded proteins such as Bid and Bax, and the other one is apoptotic suppressing gene-encoded proteins such as Bcl-2 and Bcl-XL. ${ }^{15-18}$ These proteins play important roles in the process of apoptosis. As mitochondria play a major steplimiting role in apoptosis of cells, ${ }^{12}$ it is necessary to deliver drugs to the mitochondria in order to activate pro-apoptotic genes or inhibit apoptotic suppressing genes.

Proposed here is a new strategy to circumvent the MDR of leukemia by designing cancer cell mitochondriaspecific drug-loaded liposomes, ie, antiresistant epirubicin mitosomes. In the lipid-based mitosomes, epirubicin was encapsulated into the vesicle as an anticancer agent, amlodipine was simultaneously incorporated into the vesicle as an apoptotic inducer of leukemia, and dequalinium was inserted into the lipid bilayer membrane of the mitosome as a mitochondrial targeting molecule. Antiresistant epirubicin mitosomes were developed for the following purposes: (1) the resistant/nonresistant leukemia cells could be killed directly by antiresistant epirubicin mitosomes; (2) the mitochondrial genes could be regulated by apoptotic inducer amlodipine, which was selectively delivered to the mitochondria by the antiresistant epirubicin mitosomes.

Epirubicin is an anthracycline drug used for chemotherapy. It acts by intercalating with DNA strands and triggering DNA cleavage via topoisomerase II, resulting in death of cancer cells. In addition, epirubicin generates free radicals that cause cell and DNA damage. ${ }^{19}$ Amlodipine is a dihydropyridine $\mathrm{Ca}^{2+}$ channel blocker used for treating hypertension. In the present study, amlodipine was used as a pro-apoptotic inducer of leukemia because it was previously found that amlodipine was able to potentiate the apoptosis of leukemia cells. ${ }^{20}$ Dequalinium is a dicationic compound resembling "bola"-form electrolytes, and a symmetrical molecule with two charge centers separated at a relatively large distance. It is able to accumulate in the mitochondria of living cells in response to mitochondrial membrane potential. ${ }^{21,22}$ Dequalinium was thus modified on the mitosomes as a specific ligand for targeting mitochondria of leukemia cells.

In the present study, the building of antiresistant epirubicin mitosomes was based on the following hypotheses: antiresistant epirubicin mitosomes may enter into the cancer cells by phagocytosis and remain as two forms: namely, the integrated mitosomes and the ruptured mitosomes. The integrated mitosomes can target mitochondria due to mitochondrial affinity of dequalinium modified on the mitosomes. ${ }^{23}$ Accordingly, epirubicin and amlodipine may activitate the Bax/Bak channel, induce the PT pore opening, damage the membrane of mitochondria, and thus result in the release of apoptogenic factors like cytochrome $\mathrm{c}$ and apoptosis-inducing factor (AIF). The degradation of nuclear DNA caused by the released AIF is not via the caspase pathway. On the contrary, released cytochrome c triggers the apoptosis pathway depending on the activating caspase family, including binding with apoptosis protease activating factor (Apaf)-1 and then stimulating caspase-9. The cleavage of caspase- 9 initiates a cascade of caspase activation like caspase-3, leading to apoptosis of cancer cells. ${ }^{24}$ On the other hand, the mitosomes may induce apoptosis by inhibiting $\mathrm{Bcl}-2$ and $\mathrm{Bcl}-\mathrm{XL}$ in mitochondria. Furthermore, epirubicin 
from the ruptured mitosomes may intercalate DNA strands in the nucleus and directly result in the death of cancer cells.

The objectives of the present study were to prepare the antiresistant epirubicin mitosomes and characterize their properties on human chronic myelogenous leukemia K562 and MDR K562/ADR cells in vitro and in vivo.

\section{Design and methods Preparation of antiresistant epirubicin mitosomes}

Four types of liposomes were prepared: (1) antiresistant epirubicin mitosomes, which were used as the cancer cell mitochondria-specific liposomes; (2) antiresistant epirubicin liposomes; (3) epirubicin liposomes; and (4) amlodipine liposomes. The latter three types of liposomes were used as the controls.

\section{Antiresistant epirubicin mitosomes}

Egg phosphatidylcholine (EPC), 3 $\beta-\left[\mathrm{N}-\left(\mathrm{N}^{\prime}, \mathrm{N}^{\prime}-\right.\right.$ dimethylaminoethane)-carbamoyl] cholesterol hydrochloride (DC-cholesterol HCl; Avanti Polar Lipids, Alabaster, AL), polyethylene glycol-distearoylphosphosphatidyl ethanolamine (PEG2000-DSPE; NOF Corporation, Tokyo, Japan), and dequalinium (Hangzhou Sanhe Chemicals Co, Ltd, Hangzhou, China) (in a 60:25:5:15 $\mu \mathrm{mol}$ ratio) were dissolved in methanol in a pear-shaped flask. The methanol was evaporated to dryness under vacuum with a rotary evaporator, and then the formed lipid film was hydrated with $250 \mathrm{mM}$ ammonium sulfate by sonication in the water bath for 5 minutes and followed by sonication using a probe-type sonicator for 6.8 minutes. The suspensions after hydration were successively extruded through polycarbonate membranes (Millipore, Bedford, MA), with pore sizes of 400 and $200 \mathrm{~nm}$, three times, respectively. The blank liposomes modified with dequalinium were then obtained and dialyzed (12,000-14,000 molecular mass cutoff) in phosphate-buffered saline (PBS) (137 mM NaCl, $2.7 \mathrm{mM}$ $\mathrm{KCl}, 8 \mathrm{mM} \mathrm{Na}_{2} \mathrm{HPO}_{4}$, and $2 \mathrm{mM} \mathrm{KH}_{2} \mathrm{PO}_{4}, \mathrm{pH}$ 7.4) three times ( 12 hours each time). Epirubicin and amlodipine were loaded using an ammonium sulfate gradient loading method, as reported previously. ${ }^{20}$ The blank liposomes modified with dequalinium were mixed with an appropriate amount of epirubicin hydrochloride (Nanjing Tianzun Zezhong Chemicals Ltd, Jinagsu, China), incubated at $60^{\circ} \mathrm{C}$ in a water bath, and intermittently shaken for 20 minutes. An appropriate amount of amlodipine besylate (Beijing Yimin Pharmaceuticals Co, Ltd, Beijing, China) was added to the above liposomes, further incubated for 10 minutes, producing the antiresistant epirubicin mitosomes.

\section{Antiresistant epirubicin liposomes}

The antiresistant epirubicin liposomes were prepared using the same procedure used to prepare the antiresistant epirubicin mitosomes, excluding the addition of dequalinium in the formed lipid membrane.

\section{Epirubicin liposomes}

The epirubicin liposomes were prepared using the same procedure used to prepare the antiresistant epirubicin liposomes, excluding the addition of amlodipine during drug loading.

\section{Amlodipine liposomes}

The amlodipine liposomes were prepared using the same procedure used to prepare the antiresistant epirubicin liposomes, excluding the addition of epirubicin during drug loading. The incubation duration for loading amlodipine was 30 minutes.

\section{Characterization of the liposomes}

Epirubicin, amlodipine, and dequalinium were measured on ODS column (Nucleodur 100-5 C18, $250 \mathrm{~mm} \times 4.6 \mathrm{~mm}$, $5 \mu \mathrm{m}$ ) by high performance liquid chromatography (HPLC) system with ultraviolet detector (Agilent Technologies Inc, Cotati, CA). The mobile phase consisted of acetonitrile, $0.02 \mathrm{M} \mathrm{NaH}_{2} \mathrm{PO}_{4}$ and triethylamine (34.0:66.0:0.3, v/v) and adjusted to $\mathrm{pH} 4.0$ with phosphoric acid. The detection wavelength was set at $240 \mathrm{~nm}$, the flow rate was $1.0 \mathrm{~mL} / \mathrm{min}$, and the injection volume was $20 \mu \mathrm{L}$.

All the liposomes were passed over a Sephadex G-50 column (Sigma-Aldrich Corporation agent, Beijing, China) to remove the unencapsulated epirubicin and amlodipine or unmodified dequalinium. The encapsulation efficiency of epirubicin or amlodipine was calculated with the formula: $\mathrm{EE}=\mathrm{W}_{\text {encap }} / \mathrm{W}_{\text {total }} \times 100 \%$, where EE is the encapsulation efficiency of epirubicin or amlodipine, $\mathrm{W}_{\text {encap }}$ is the measured amount of epirubicin or amlodipine in the liposome suspensions after passing over the column, and $\mathrm{W}_{\text {total }}$ is the measured amount of epirubicin or amlodipine in the equal volume of liposome suspensions before passing over the column. The modifying efficiency of dequalinium was defined as $\mathrm{W}_{\text {deq }}$ per $\mu$ mol lipids, where $\mathrm{W}_{\text {deq }}$ is the measured amount of dequalinium on the liposome surface. The liposome sample was destroyed and measured by HPLC as above. 
The particle sizes, polydispersity indexes (PDI), and zeta potential values of all liposomes were measured with Zetasizer 3000HSA (Malvern Instruments Ltd, Worcestershire, UK). The antiresistant epirubicin mitosomes were stained with $1 \%$ uranyl acetate and observed under transmission electron microscopy (TEM) (Tecnai G2 20ST, FEI Co, Tokyo, Japan).

In-vitro drug release assays of epirubicin and amlodipine from all liposomes were performed using the method reported previously. ${ }^{25} \mathrm{~A}$ volume of $2 \mathrm{~mL}$ antiresistant epirubicin mitosomes, antiresistant epirubicin liposomes, epirubicin liposomes, or amlodipine liposomes was mixed with $2 \mathrm{~mL}$ of release medium (a mixture of PBS and 10\% fetal bovine serum $[\mathrm{FBS}]$ ), and the mixture was then placed into the dialysis tubing. The dialysis tubing was placed into $20.0 \mathrm{~mL}$ of the release medium, and oscillated with a shaker at a rate of 100 times per minute at $37^{\circ} \mathrm{C}$. A volume of $0.2 \mathrm{~mL}$ release medium was sampled at $0,0.25,0.50,1.00,2.00,4.00,8.00$, $12.00,24.00$, and 48.00 hours, followed by immediately adding an equal volume of fresh release medium. The content of epirubicin or amlodipine was determined by HPLC as above, and the release rate was estimated with the formula: $\mathrm{RR}=\left(\mathrm{W}_{\mathrm{i}} / \mathrm{W}_{\text {total }}\right) \times 100 \%$, where $\mathrm{RR}$ is the drug release rate $(\%), \mathrm{W}_{\mathrm{i}}$ is the measured drug amount of each sample, and $\mathrm{W}_{\text {total }}$ is the total drug amount in an equal volume of liposome suspension before performing the release experiment.

\section{Cells and cell culture}

Human chronic myelogenous leukemia (K562) and multidrug resistant human chronic myelogenous leukemia (MDR K562/ ADR cells) (Institute of Hematology, Chinese Academy of Medical Sciences and Peking Union Medical College, Tianjin, China) were used. The culture medium was prepared with RPMI (Roswell Park Memorial Institute) 1640 (Macgene Biotech Co, Ltd, Beijing, China) supplemented with 12\% heat-inactivated FBS (Invitrogen Corporation, New York, NY), antibiotics (penicillin $100 \mathrm{U} / \mathrm{mL}$, streptomycin $100 \mu \mathrm{g} / \mathrm{mL}$ ), and $2 \mathrm{mM} \mathrm{L}$-glutamine. The cell culture was performed in the incubator $\left(37^{\circ} \mathrm{C}\right.$ and $\left.5 \% \mathrm{CO}_{2}\right)$.

\section{Cytotoxicity assay}

Microtiter tetrazolium (MTT) assay was performed according to a standard MTT-based colorimetric assay. ${ }^{26}$ Briefly, K562, or MDR K562/ADR cells were seeded into a 96-well plate at $2 \times 10^{4}$ cells per well, and the cells were cultured in the incubator for 24 hours. Fresh medium containing serial concentrations of various drug formulations was then added into the plate well, including free epirubicin, free amlodipine, mixture of various concentrations of free epirubicin and amlodipine, epirubicin liposomes, amlodipine liposomes, antiresistant epirubicin liposomes, antiresistant epirubicin mitosomes, and PBS-loaded mitosomes. Cells incubated in medium without any drug were used as controls. After treatment, cells were incubated for 43 hours, and then $20 \mu \mathrm{L} /$ well 3-(4,5-dimethylthiazol-2-yl)-2,5-diphenyl tetrazolium bromide $(5 \mathrm{mg} / \mathrm{mL})$ was added. The plates were incubated for an additional 5 hours. The cells were then lyzed using $150 \mu \mathrm{L}$ of sodium dodecyl sulfate (SDS) solution (10 g SDS, $5 \mathrm{~mL}$ iso-butyl alcohol, $10 \mathrm{~mL} 0.12 \mathrm{~mol} / \mathrm{L} \mathrm{HCl}$, deionized water made up to $100 \mathrm{~mL}$ ) and placed overnight in the incubator at $37^{\circ} \mathrm{C}$. The absorbance values of the lyzed cells were read on a microplate reader (Model 680; BIO-RAD Laboratories, Tokyo, Japan) at a wavelength of $490 \mathrm{~nm}$. The survival rates were calculated with the formula: survival rate $(\%)=\left(\mathrm{A}_{490 \mathrm{~nm}}\right.$ treated cells $/ \mathrm{A}_{490 \mathrm{~nm}}$ control cells) $\times 100 \%$, where $\mathrm{A}_{490 \mathrm{~nm}}$ is the absorbance value at $490 \mathrm{~nm}$.

\section{Mitochondrial targeting}

A confocal laser scanning fluorescent microscope with Leica confocal software (Leica, Heidelberg, Germany) was used to observe the mitochondrial targeting properties of antiresistant epirubicin mitosomes. Briefly, K562 or MDR K562/ADR cells were seeded into 6 -well plates at $1 \times 10^{6}$ cells per well. After incubation for 24 hours, drugs were added, respectively, including free epirubicin $(10 \mu \mathrm{M})$, epirubicin liposomes $(10 \mu \mathrm{M})$, antiresistant epirubicin liposomes $(10 \mu \mathrm{M}$ for epirubicin or for amlodipine), and antiresistant epirubicin mitosomes (10 $\mu \mathrm{M}$ for epirubicin or for amlodipine). After treatment, cells were incubated for 1 hour, and then the cells were washed with PBS and stained with MitoTracker ${ }^{\circledR}$ Green FM (InvivoGen Corporation agent, Beijing, China). Composite images were made by overlapping the images of the individual channels.

\section{Caspase- 9 and -3 activity assays}

The activity of caspase-9 was measured using caspase-9 colorimetric assay kit (Nanjing KeyGen Biotech Co, Ltd, Nanjing, China). Briefly, K562 or MDR K562/ADR cells were cultured for 24 hours. Drugs were added, respectively, including free epirubicin $(10 \mu \mathrm{M})$, free amlodipine $(10 \mu \mathrm{M})$, epirubicin liposomes $(10 \mu \mathrm{M})$, antiresistant epirubicin liposomes (10 $\mu \mathrm{M}$ for epirubicin or for amlodipine) and antiresistant epirubicin mitosomes $(10 \mu \mathrm{M}$ for epirubicin or for amlodipine). The cells were further incubated for 12 hours, collected, and washed twice with PBS by centrifugation at 2000 revolutions per minute (rpm) for 5 minutes. Cold lysis 
buffer containing dithiothreitol was then added into the collected cells and mixed homogeneously. The cells were incubated on ice for 60 minutes and then vortexed three or four times for 10 seconds each time. The cell lysates were then centrifuged at 10,000 rpm for 1 minute at $4^{\circ} \mathrm{C}$. Finally, the supernatants were sucked off and transferred to new tubes on ice. The assay for caspase- 9 was based on spectrophotometric detection of the chromophore $p$-nitroanilide ( $p$ NA) after cleavage from the labeled substrate LEHD- $p$ NA. The $p$ NA light emission was quantified using a microplate reader at $405 \mathrm{~nm}$. Caspase- 9 activity was calculated with the formula: caspase- 9 activity $=$ (caspase- 9 protein level of the treated cells)/(caspase-9 protein level of the control cells).

Caspase-3 activity was measured using the caspase-3 colorimetric assay kit (Nanjing KeyGen Biotech Co, Ltd, Nanjing, China), and the procedure was the same as for measuring caspase-9 activity. The assay is based on spectrophotometric detection of the chromophore $p$ NA after cleavage from the labeled substrate DEVD- $p \mathrm{NA}$. The $p \mathrm{NA}$ light emission was quantified using a microplate reader at $405 \mathrm{~nm}$. Caspase- 3 activity was calculated with the formula: caspase- 3 activity $=($ caspase -3 protein level of the treated cells)/(caspase-3 protein level of the control cells).

\section{In-vivo antitumor effects and TUNEL (terminal deoxynucleotidyl transferase dUTP nick end labeling) analysis}

All animal experiments were adhered to the principles of care and use of laboratory animals and were approved by the Institutional Animal Care and Use Committee of Peking University. Female BALB/c nude mice (initially weighing 14-16 g, Peking University Health Science Center) were divided into five groups (six in each). Cyclophosphamide was injected intraperitoneally into the mice at a dose of $2 \mathrm{mg}$ per mouse per day for two consecutive days. Approximately $2.0 \times 10^{7}$ MDR K562/ADR cells re-suspended in $200 \mu \mathrm{L}$ of serum-free cell culture medium were subcutaneously injected into the right flanks of the nude mice. On day 7, 9, 11, 13, 15 , and 17 after inoculation, physiological saline, free epirubicin (3 mg/kg), epirubicin liposomes ( $3 \mathrm{mg} / \mathrm{kg}$ epirubicin), antiresistant epirubicin liposomes $(3 \mathrm{mg} / \mathrm{kg}$ epirubicin plus $1.2 \mathrm{mg} / \mathrm{kg}$ amlodipine), and antiresistant epirubicin mitosomes ( $3 \mathrm{mg} / \mathrm{kg}$ epirubicin plus $1.2 \mathrm{mg} / \mathrm{kg}$ amlodipine) were injected into mice via the tail vein. The tumor sizes of the mice were monitored once daily, and tumor volumes were calculated as length $\times$ width ${ }^{2} \times 0.52\left(\mathrm{~mm}^{3}\right) .{ }^{27}$

Tumor-bearing mice were sacrificed on day 20 by cervical dislocation. Tumor masses were carefully isolated, and fixed for analysis using TUNEL. An ApopTag plus peroxidase in-situ apoptosis detection kit (Intergen Co, Ltd, Burlington, MA) was used to visualize the cells with DNA fragmentation, as reported previously. ${ }^{28,29}$ Nonnecrotic zone was selected in the tumor section, and images were randomly selected under the light microscope. In the presence of apoptosis, the apoptotic cells manifested as brownish staining in the nuclei. At least 1000 tumor cells were counted, and the percentage of TUNEL-positive cells was determined.

\section{Statistics}

Data are presented as means \pm standard deviations. One-way analysis of variance was used to determine significance among groups, after which post-hoc tests with the Bonferroni correction were used for comparison between individual groups. A value of $P<0.05$ was considered to be significant.

\section{Results}

\section{Characterization of the mitosomes}

For antiresistant epirubicin mitosomes, antiresistant epirubicin liposomes, epirubicin liposomes, and amlodipine liposomes, the encapsulation efficiencies of epirubicin or amlodipine were all $\geq 90 \%$. For antiresistant epirubicin mitosomes, the modifying efficiency of dequalinium was approximately $70 \mu \mathrm{g} / \mu \mathrm{mol}$ lipids. For antiresistant epirubicin mitosomes, antiresistant epirubicin liposomes, epirubicin liposomes, and amlodipine liposomes, the mean particle sizes were $109.2 \pm 0.8,107.8 \pm 1.4,113.7 \pm 1.4$, and $112.6 \pm 5.7 \mathrm{~nm}$, respectively. The particle size distributions (PDI) were $0.196 \pm 0.002,0.222 \pm 0.006,0.253 \pm 0.007$, and $0.251 \pm 0.026$, respectively. The zeta potential values were $1.564 \pm 0.370,1.129 \pm 0.223,0.900 \pm 0.450$, and $0.666 \pm 0.169 \mathrm{mV}$, respectively.

For antiresistant epirubicin mitosomes, antiresistant epirubicin liposomes, and epirubicin liposomes in vitro, the release rates of epirubicin at 48 hours were $10.36 \% \pm 0.19 \%$, $30.42 \% \pm 0.97 \%$, and $21.38 \% \pm 0.14 \%$, respectively (Figure 1A). For antiresistant epirubicin mitosomes, antiresistant epirubicin liposomes, and amlodipine liposomes in vitro, the release rates of amlodipine at 48 hours were $24.41 \% \pm 0.17 \%, 38.57 \% \pm 0.63 \%$, and $14.65 \% \pm 0.22 \%$, respectively (Figure 1B).

Figure 1C shows the TEM image of antiresistant epirubicin mitosomes. Results showed that antiresistant epirubicin mitosomes were monodispersed and spherical with a diameter between 100 and $110 \mathrm{~nm}$. 

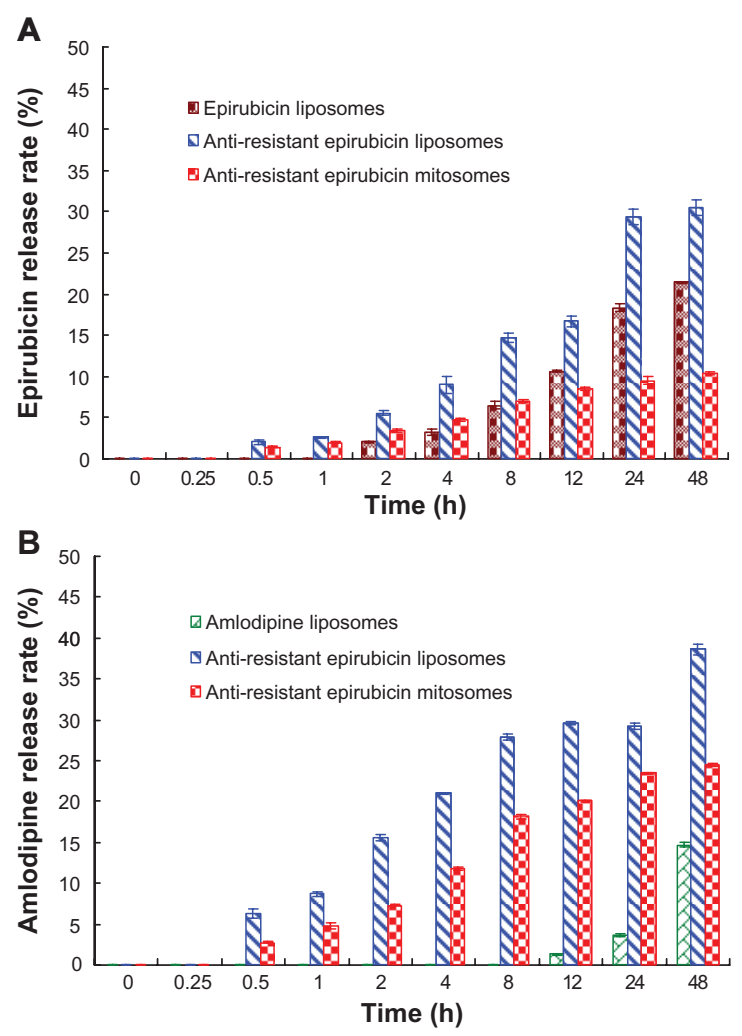

C

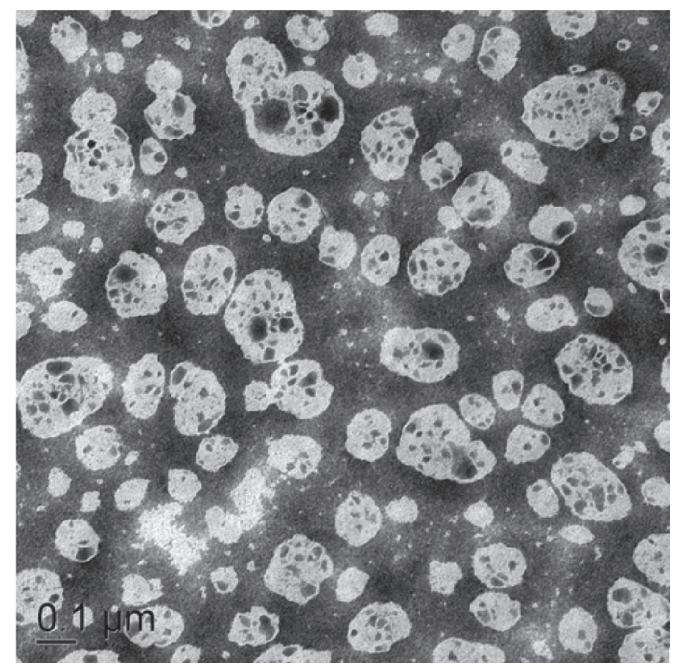

Figure I (A) In-vitro release rates (\%) of epirubicin from epirubicin liposomes, amlodipine liposomes, anti-resistant epirubicin liposomes, and anti-resistant epirubicin mitosomes in PBS containing $10 \% \mathrm{FBS}$ at $37^{\circ} \mathrm{C}$. (B) In-vitro release rates (\%) of amlodipine from epirubicin liposomes, amlodipine liposomes, anti-resistant epirubicin liposomes, and anti-resistant epirubicin mitosomes in PBS containing $10 \% \mathrm{FBS}$ at $37^{\circ} \mathrm{C}$. Data are presented as means \pm standard deviations $(n=3)$. (C) Transmission electron microscopy image of anti-resistant epirubicin mitosomes. Note: Scale bar $=100 \mathrm{~nm}$.

Abbreviations: PBS, phosphate-buffered saline; FBS, fetal bovine serum.

\section{Cytotoxicity}

Figure 2Ai and Aii show the MTT assay results after epirubicin alone or epirubicin plus amlodipine were applied to K562 cells and MDR K562/ADR cells, respectively. Epirubicin alone $(0.05-2.0 \mu \mathrm{M})$ was effective in inhibiting the proliferation of K562 cells, showing a dose-dependent manner. After the addition of amlodipine $(10$ or $20 \mu \mathrm{M})$, the inhibitory rates of epirubicin $(0.05-2.0 \mu \mathrm{M})$ to $\mathrm{K} 562$ cells were significantly increased, displaying an evident synergistic effect. The inhibitory rates to MDR K562/ADR cells of epirubicin were $<20 \%$ at a concentration ranging from 0.05 to $2.0 \mu \mathrm{M}$. After co-treating various concentrations of epirubicin $(0.05-2.0 \mu \mathrm{M})$ with a fixed concentration of amlodipine $(5.0,10.0$, or $20.0 \mu \mathrm{M})$, the survival rates of MDR K562/ADR cells were significantly reduced, compared with those treated with epirubicin alone at the same concentration. The inhibiting effect was increased with the rise of amlodipine concentration, displaying a dosedependent manner.

Figure 2Bi and Bii depict the effects on the proliferation of K562 and MDR K562/ADR cells after applying free epirubicin, free amlodipine, epirubicin liposomes, amlodipine liposomes, antiresistant epirubicin liposomes, and antiresistant epirubicin mitosomes. Results showed that antiresistant epirubicin mitosomes exhibited the strongest inhibitory effect to the proliferation among all the groups in both K562 cells and MDR K562/ADR cells. The inhibiting effect to the cells was increased with the rise of epirubicin or amlodipine concentration in antiresistant epirubicin mitosomes, displaying a dose-dependent manner.

Figure 2C shows the growth inhibition effect on K562 and MDR K562/ADR cells of PBS-loaded mitosomes. The survival rates of K562 and MDR K562/ADR cells after applying PBS-loaded mitosomes for 43 hours were $87.10 \% \pm 5.10 \%$ and $68.65 \% \pm 9.14 \%$, respectively, where the membrane components were equivalent to antiresistant epirubicin mitosomes loaded with $10 \mu \mathrm{M}$ epirubicin.

\section{Mitochondria-targeting by confocal assay}

Figure 3A-F, M, G-L, and N represent the confocal laser scanning microscopic images of K562 and MDR K562/ ADR cells after applying drugs to the cells for 1 hour at $37^{\circ} \mathrm{C}$ and stained with MitoTracker Green, respectively. Figure 3C-F and $\mathrm{M}$ represent the confocal laser scanning microscopic images after applying free epirubicin, epirubicin liposomes, antiresistant epirubicin liposomes, and antiresistant epirubicin mitosomes to K562 cells, respectively. Similarly, Figure 3I-L and N denote the results after applying the above drugs to MDR K562/ADR cells, respectively.

In the images, mitochondria were seen as the green fluorescent color stained by MitoTracker Green (Figure 3B, Ci, Di, Ei, Fi, Mi; Figure 3H, Ii, Ji, Ki, Li, and Ni), and epirubi- 

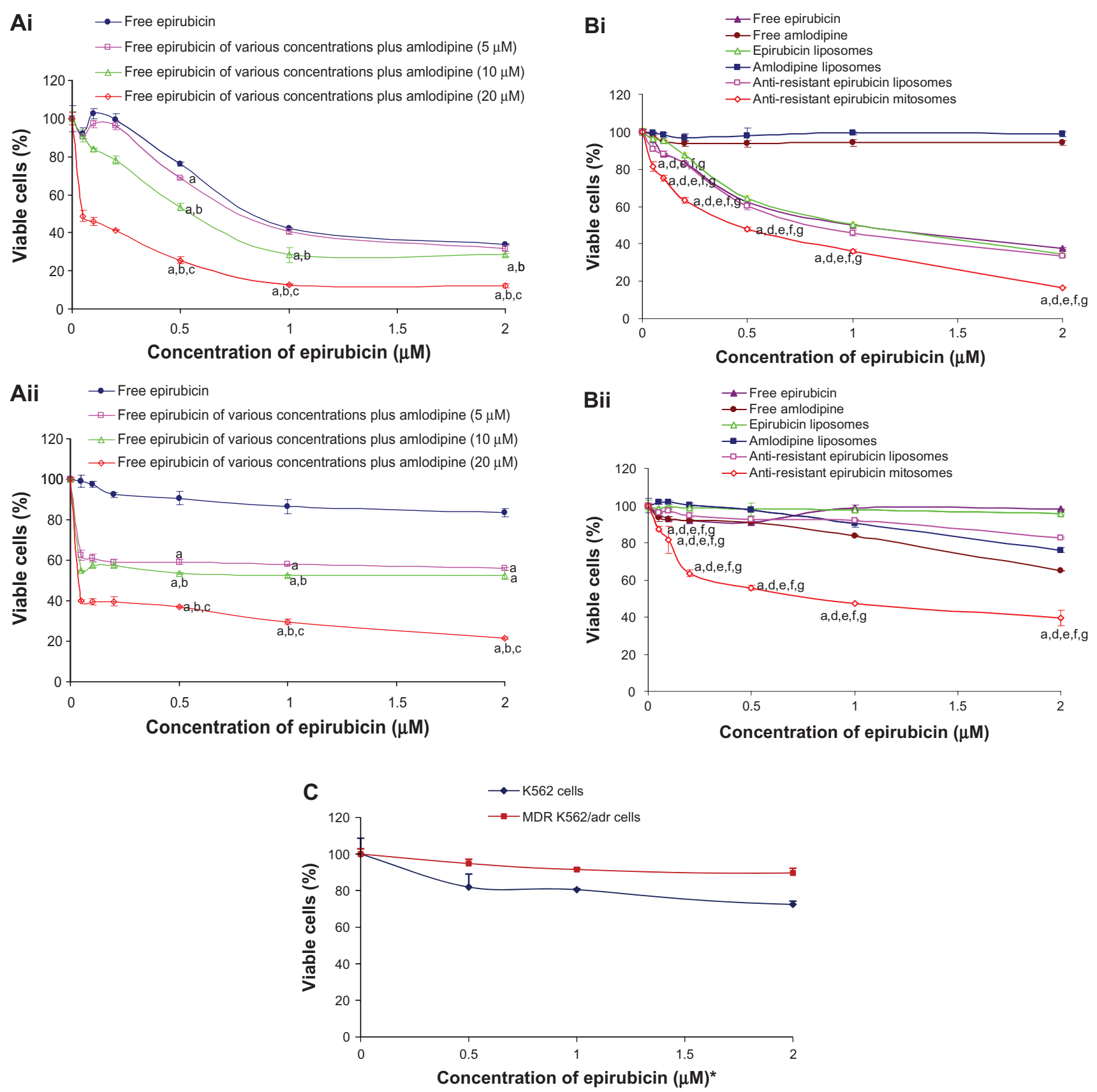

Figure 2 (A) Effects of free epirubicin, free amlodipine, or free epirubicin co-treated with free amlodipine on the viable rates of K562 (i) and MDR K562/ADR cells (ii) measured by microtiter tetrazolium assay. (B) Effects of various formulations on the viable rates of K562 (i) and MDR K562/ADR cells (ii). (C) Effects of phosphate-buffered saline-loaded mitosomes on the viable rates of K562 and K562/ADR cells.

Notes: ${ }^{a} P<0.05$, versus free epirubicin; ${ }^{b} P<0.05$, versus free epirubicin of various concentrations plus amlodipine $(5.0 \mu M)$; ${ }^{c} P<0.05$, versus free epirubicin of various concentrations plus amlodipine $(10.0 \mu \mathrm{M})$; ${ }^{\mathrm{d}} P<0.05$, versus free amlodipine; ${ }^{\mathrm{e} P}<0.05$, versus epirubicin liposomes; ${ }^{f} P<0.05$, versus amlodipine liposomes; ${ }^{8} P<0.05$, versus epirubicin plus amlodipine liposomes; *the membrane components were comparable to those of anti-resistant epirubicin mitosomes at the designated concentration point but not containing drugs in the empty mitosomes. Data are presented as the means \pm standard deviations $(n=3)$.

Abbreviation: MDR, multidrug resistant.

cin from free epirubicin, epirubicin liposomes, antiresistant epirubicin liposomes, or antiresistant epirubicin mitosomes was shown as a red fluorescent color (Figure 3Cii, Dii, Eii, Fii, and Mii; Figure 3Iii, Jii, Kii, Lii, and Nii). A bright yellow fluorescent color represented a composition of red and green fluorescence, demonstrating that the liposomes containing epirubicin were co-localized into mitochondria (Figure 3Ciii, Diii, Eiii, Fiii, and Miii; Figure 3Iiii, Jiii, Kiii, Liii, and Niii).

The results show that free epirubicin, epirubicin liposomes, and antiresistant epirubicin liposomes mostly distributed in the nucleus of the K562 cells and 

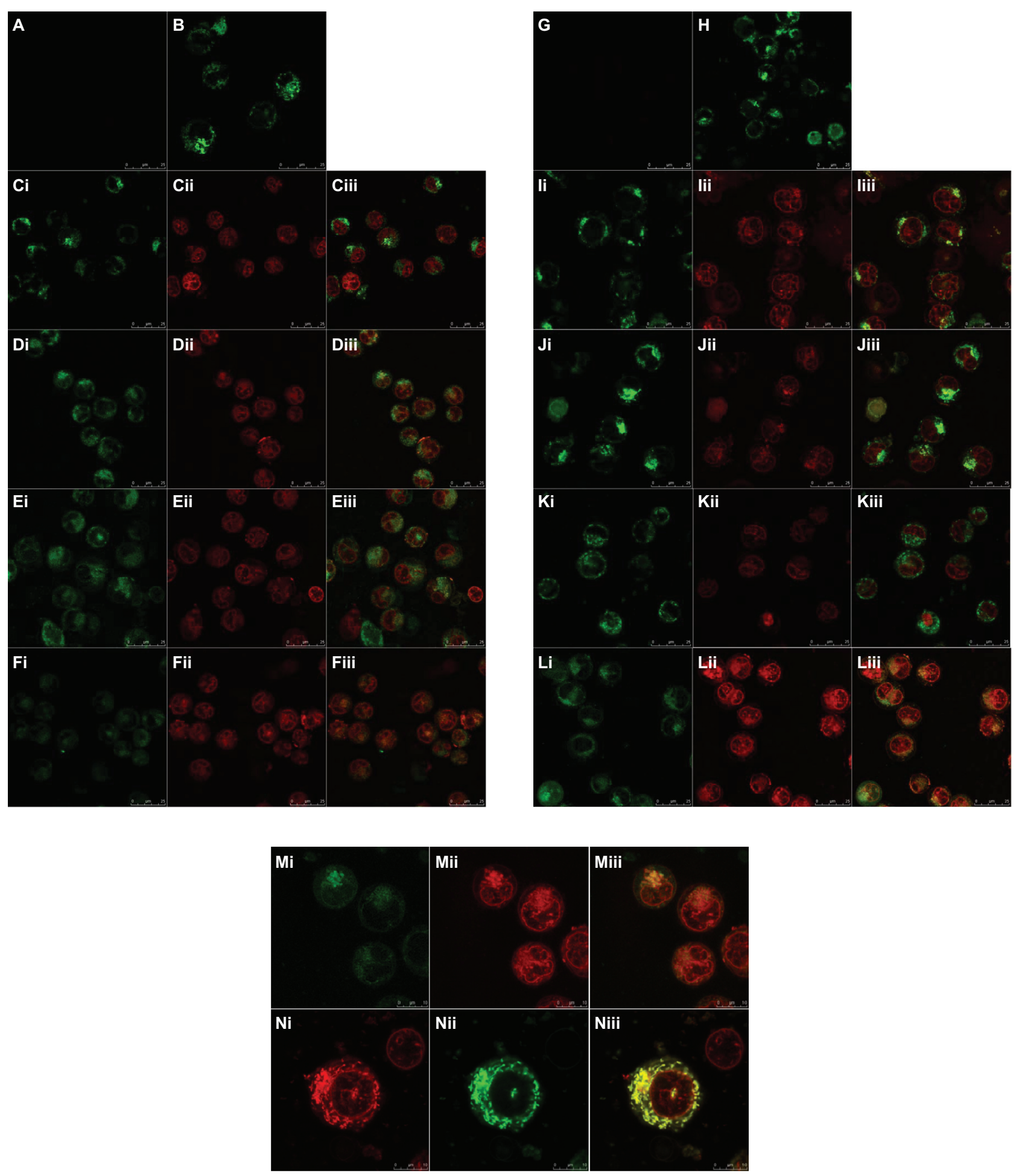

Figure 3 (A-F, M) Laser confocal fluorescence images of K562 cells incubated with various formulations for I hour at $37^{\circ} \mathrm{C}$ (magnification $\times 200$ or $\times 600$ ). (G-L, N) Laser confocal fluorescence images of MDR K562/ADR cells incubated with various formulations for I hour at $37^{\circ} \mathrm{C}$ (magnification $\times 200$ or $\times 600$ ). (A and $\left.\mathbf{G}\right)$ Treated with PBS as a blank control. (B and $\mathbf{H})$ Treated with PBS and stained with MitoTracker Green. (C and I) Treated with free epirubicin (I0 $\mu$ M) and stained with MitoTracker Green. (D and J) Treated with epirubicin liposomes (10 $\mu$ M epirubicin) and stained with MitoTracker Green. (E and K) Treated with anti-resistant epirubicin liposomes (10 $\mu$ M epirubicin plus I0 $\mu$ M amlodipine) and stained with MitoTracker Green. (F, L, $\mathbf{M}$ and $\mathbf{N}$ ) Treated with anti-resistant epirubicin mitosomes (I0 $\mu$ M epirubicin plus I0 $\mu$ M amlodipine) and stained with MitoTracker Green.

Notes: (i) Green channel: MitoTracker Green ${ }^{\circledR}$ (InvivoGen Corporation agent, Beijing, China) stained mitochondria. (ii) Red channel: epirubicin. (iii) Composite images of (i) and (ii). The bright yellow fluorescence in Fiii, Liii, Miii, and Niii shows that anti-resistant epirubicin mitosomes are co-localized into the mitochondria of both K562 and MDR K562/ADR cells, demonstrating that the anti-resistant epirubicin mitosomes are selectively accumulated into the mitochondria of both leukemia cells.

Abbreviations: MDR, multidrug resistant; PBS, phosphate-buffered saline. 
MDR K562/ADR cells (Figure 3D-E; Figure 3I-K). On the contrary, antiresistant epirubicin mitosomes selectively accumulated into the mitochondria of both the K562 cells (Figure 3F and M) and MDR K562/ADR cells (Figure $3 \mathrm{~L}$ and $\mathrm{N}$ ).

\section{Caspase- 9 and -3 activity}

Figure 4A and B show the results of caspase- 9 activity assays and caspase- 3 activity assays in K562 cells and MDR K562/ ADR cells, respectively. Results from caspase- 9 assay showed that the activity of caspase-9, in both K562 and MDR K562/ ADR cells, was significantly increased by antiresistant epirubicin mitosomes, compared with that of control. The results in K562 cells indicated that the caspase- 9 activity of K562 cells was increased after adding free epirubicin, free amlodipine, epirubicin liposomes, antiresistant epirubicin liposomes, or antiresistant epirubicin mitosomes. The caspase-9 activity of K562 cells after treating with antiresistant epirubicin mitosomes
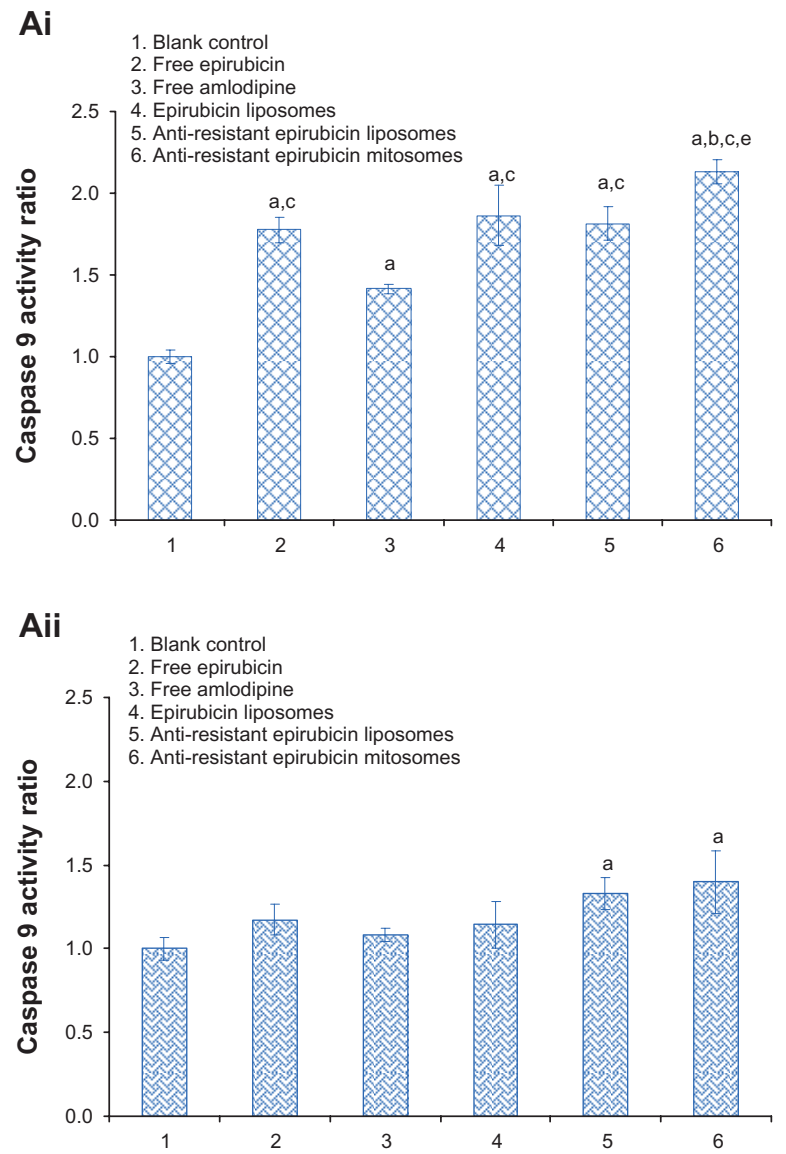

was the highest among all the treatment groups (Figure 4Ai). The results in MDR K562/ADR cells indicated that the caspase-9 activity of MDR K562/ADR cells was increased after adding antiresistant epirubicin liposomes or antiresistant epirubicin mitosomes. The caspase-9 activity of MDR K562/ ADR cells was slightly higher after treating with antiresistant epirubicin mitosomes compared with that after treating with antiresistant epirubicin liposomes (Figure 4Aii).

Results from caspase-3 assay in K562 and MDR K562/ ADR cells showed a similar result to that of caspase- 9 (Figure 4Bi and Bii).

\section{In-vivo antitumor effects and TUNEL analysis}

The cancer masses appeared at the 5th or 6th day after MDR K562/ADR cells were inoculated in the nude mice. Compared with the control group administered as physiological saline, the tumor growth was obviously inhibited in all treatment
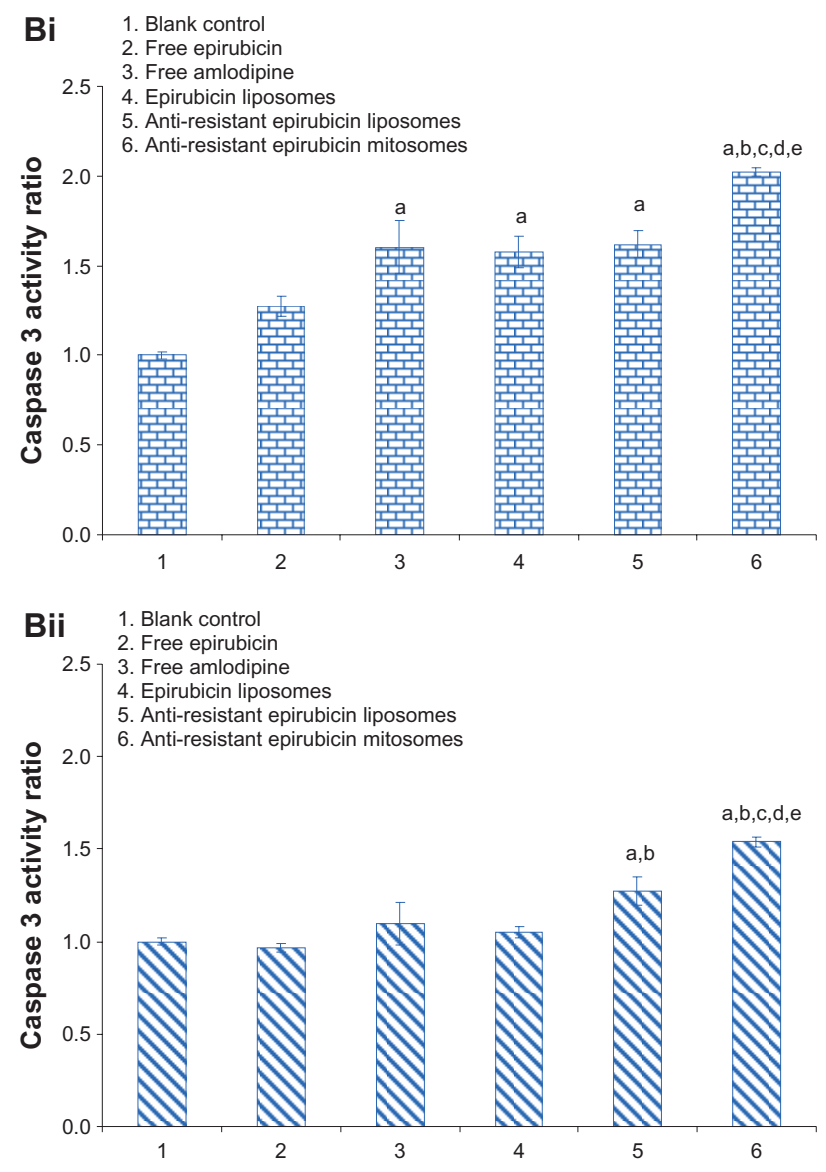

Figure 4 (A) Caspase-9 activity in K562 (Ai and Bi) and MDR K562/ADR cells (Aii and Bii) induced by various formulations. (B) Caspase-3 activity in K562 (Ai and Bi) and MDR K562/ADR cells (Aii and Bii) induced by various formulations.

Notes: Data are presented as means \pm standard deviations $(n=3)$. ${ }^{a} P<0.05$, versus the blank control; ${ }^{b} P<0.05$, versus free epirubicin; ${ }^{c} P<0.05$, versus free amlodipine; ${ }^{d} P<0.05$, versus epirubicin liposomes; ${ }^{e} P<0.05$, versus antiresistant epirubicin liposomes.

Abbreviation: MDR, multidrug resistant. 
groups, but the antitumor effect varied. After giving antiresistant epirubicin mitosomes, the tumor volumes from the 8 th to the 13 th day were significantly smaller compared with those after administering free epirubicin alone, and at the 20th day were significantly smaller compared with those after injecting free epirubicin alone, epirubicin liposomes, or antiresistant epirubicin liposomes. In comparison among groups, the rank order for antitumor effect in vivo was as follows: antiresistant epirubicin mitosomes $>$ antiresistant epirubicin liposomes $>$ epirubicin liposomes $>$ free epirubicin alone $>$ physiological saline (the control) (Figure 5A). Results showed that the treatment of antiresistant epirubicin mitosomes resulted in a robust efficacy in reducing the tumor volume.

The TUNEL assay showed that the apoptotic percentages were $7.2 \% \pm 0.8 \%$ for antiresistant epirubicin mitosomes (Figure $5 \mathrm{Bv}$ ), $4.8 \% \pm 1.0 \%$ for antiresistant epirubicin liposomes (Figure 5Biv), 3.0\% $\pm 0.6 \%$ for epirubicin liposomes (Figure $5 \mathrm{Biii}$ ), and $2.0 \% \pm 0.9 \%$ for free epirubicin (Figure 5Bii), compared with $0.5 \% \pm 0.5 \%$ for control (Figure 5Bi).

\section{Discussion}

In the present study, the mitochondrial targeting antiresistant epirubicin liposomes named as the antiresistant epirubicin mitosomes are constructed by modifying dequalinium on their surface as a mitochondrial targeting agent. Antiresistant epirubicin mitosomes show selective mitochondrial accumulation behavior and induce apoptotic effect on the resistant human chronic myelogenous leukemia. Resistant human myeloid leukemia cells are well known to be resistant to a number of pro-apoptotic agents like anticancer drugs because of the overexpression of anti-apoptosis factors, including Bcr-Abl tyrosine kinase, Bcl-2, Bcl- $\mathrm{x}_{\mathrm{L}}$, P-glycoprotein, or multidrug resistance protein. ${ }^{30-32}$ There is also a set of pro-apoptosis genes expressed in leukemia cells which can be used as the target for chemotherapy. In the membrane of cancer mitochondria, two kinds of apoptotic genes, namely apoptotic suppressing genes and pro-apoptotic genes, are associated with the drug resistance of cancer cells. ${ }^{33}$ Consequently, antiresistant epirubicin mitosomes were constructed for suppressing the anti-apoptosis genes and for activating the pro-apoptotic genes. The mitosomes were able to maintain the characteristics of the pegylated liposomes, including the long circulatory effect in the blood system. ${ }^{34}$ Therefore, antiresistant epirubicin mitosomes may provide a thorough approach for eliminating resistant and nonresistant tumors.
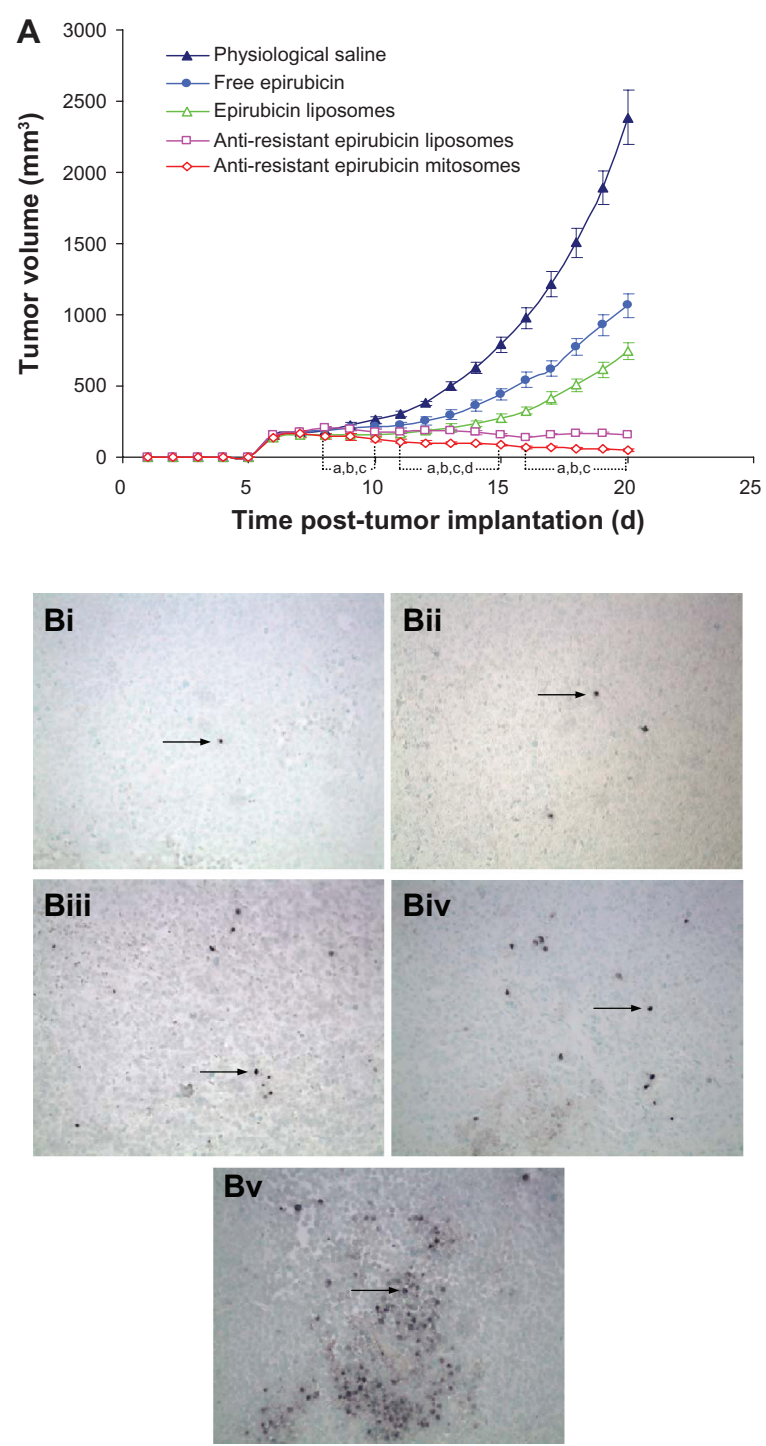

Figure 5 (A) Effect of anti-resistant epirubicin mitosomes on the MDR K562/ ADR xenografts in female nude mice. At the seventh, ninth, eleventh, thirteenth, fifteenth, and seventeeth days after inoculation, physiological saline, free epirubicin (3 $\mathrm{mg} / \mathrm{kg}$ ), epirubicin liposomes (3 $\mathrm{mg} / \mathrm{kg}$ epirubicin), anti-resistant epirubicin liposomes ( $3 \mathrm{mg} / \mathrm{kg}$ epirubicin plus $1.2 \mathrm{mg} / \mathrm{kg}$ amlodipine), and anti-resistant epirubicin mitosomes ( $3 \mathrm{mg} / \mathrm{kg}$ epirubicin plus $1.2 \mathrm{mg} / \mathrm{kg}$ amlodipine) were given to mice via tail vein, respectively. (B) Paraffin sections showing terminal deoxynucleotidyl transferase-mediated dUTP-biotin nick end-labeled cells in the tumor tissues on the 20th day after inoculation of MDR K562/ADR cells in the female nude mice (magnification $\times 200$ ). Apoptotic cells (shown by arrowhead) are characterized by a dense staining of nuclei. The MDR K562/ADR xenografted mice were given intravenously with physiological saline as a blank control (i), free epirubicin (ii), epirubicin liposomes (iii), anti-resistant epirubicin liposomes (iv), and anti-resistant epirubicin mitosomes (v).

Notes: Data are presented as the means \pm standard deviations $(n=6) .{ }^{a} p<0.05$, versus physiological saline; ${ }^{b} P<0.05$, versus free epirubicin; ${ }^{c} P<0.05$, versus epirubicin liposomes; $d P<0.05$, versus anti-resistant epirubicin liposomes.

Abbreviation: MDR, multidrug resistant.

In the present study, cytotoxicity assays were performed on human chronic myelogenous leukemia K562 and MDR K562/ADR cells to evaluate the additive antiproliferative effects of amlodipine and the antiproliferative effect of antiresistant epirubicin mitosomes, respectively. Results indicated 
that epirubicin alone is effective in inhibiting the growth of nonresistant K562 cells, but resistant to that of MDR K562/ADR cells. In the MDR K562/ADR cells, epirubicin in combination with amlodipine is effective in inhibiting the growth of MDR K562/ADR cells, indicating amlodipine was effective in reversing the resistance derived from the MDR K562/ADR cells. The reverse effect was increased with the rise in amlodipine concentration, exhibiting a dose-dependent manner. In K562 cells, the synergistic inhibitory effects are observed after co-treating with epirubicin plus amlodipine, suggesting that amlodipine was effective in enhancing the antitumor effect of epirubicin. Compared with other controls, antiresistant epirubicin mitosomes exhibited the strongest anticancer effect in K562 or MDR K562/ADR cells. After applying antiresistant epirubicin mitosomes, higher inhibition rates $(60.5 \%$ in MDR K562/ADR cells) indicated that antiresistant epirubicin mitosomes were effective in inhibiting the proliferation of MDR K562/ADR cells in vitro. The reasons were as follows: (1) apoptotic inducer amlodipine could enhance anticancer efficacy of epirubicin and reverse the resistance of MDR K562/ADR cells when epirubicin and amlodipine were simultaneously incorporated into the mitosomes; (2) antiresistant epirubicin mitosomes could enhance concentrations of the drugs accumulated in mitochondria by selectively accumulating into the mitochondria, which may enhance apoptosis of K562 and MDR K562/ADR cells.

Dequalinium is a "single-chain bala-amphiphile" 35 with two charge centers separated at a relatively large distance. Such symmetric bola-like structures are well known from archaeal lipids, which usually consist of two glycerol backbones connected by two hydrophobic chains. ${ }^{36}$ Dequalinium is able to self-associate into mitochondriotropic cationic "bola-lipid"-based vesicles, named DQAsomes. ${ }^{23,37}$ The DQAsomes have been developed for transport of drugs and DNA to mitochondria in living cells. ${ }^{38,39}$ In the present study, dequalinium is modified on the antiresistant epirubicin mitosomes with the same intention for targeting mitochondria. Mitochondria-targeting studies of antiresistant epirubicin mitosomes were performed on leukemia K562 and MDR K562/ADR cells using the confocal laser fluorescent microscopy technique. Results demonstrated that antiresistant epirubicin mitosomes were able to be colocalized into mitochondria of K562 and MDR K562/ADR cells, indicating that antiresistant epirubicin mitosomes were selectively accumulated into mitochondria of both leukemia cells, respectively.

After treating with drugs, cancer cells may be killed by injurious agents or by inducing programmed cell death (apoptosis), which may be generated by external signals (death receptor-mediated) or by internal signals (mitochondriamediated). Activation of the effector cascade differs between extrinsic and intrinsic pathways. In the intrinsic pathways mediated by mitochondria, the apoptotic inducer increases the permeability of the outer membrane, causes cytochrome $\mathrm{c}$ to be released, then results in activation of caspase- 9 and subsequent effector caspases, such as caspase-3, and finally leads to apoptosis of cells. ${ }^{40,41}$ In the present study, caspase-9 and caspase- 3 activity were measured to discover whether these pathways were involved in the process of apoptosis initiated by antiresistant epirubicin mitosomes. Results showed that, in both K562 and MDR K562/ADR cells, caspase-9 and caspase-3 activity were most evidently increased after applying antiresistant epirubicin mitosomes. It was then postulated that antiresistant epirubicin mitosomes could enter into the cancer cells, target mitochondria to induce the PT pore opening, and thereby result in the swelling of mitochondria and then spillage of cytochrome $\mathrm{c}$. The released cytochrome $\mathrm{c}$ bound with Apaf-1 in the cytosol, and changed conformation of Apaf-1, leading to subsequent bindings with pro-caspase-9 and dATP. As a result, caspase-9 was cleaved, thus initiating a cascade of caspase activation like caspase- 3 and leading to apoptosis of resistant or nonresistant leukemia cells.

Antitumor activities and apoptosis studies in vivo were performed on MDR K562/ADR cell xenografts in female BALB/c nude mice. Results showed that the antitumor effect of antiresistant epirubicin mitosomes was the most effective, compared with free epirubicin, epirubicin liposomes, or antiresistant epirubicin liposomes. TUNEL analysis demonstrated that antiresistant epirubicin mitosomes exerted favorable inducing apoptotic effect compared with other controls. The possible reasons may be as follows: (1) amlodipine as an apoptotic inducer could enhance anticancer efficacy and reverse the resistance in vivo; (2) the mitosomes as stealth liposomes with smaller particle size around $100 \mathrm{~nm}$ were able to extravasate through the "pores" of the capillary endothelium of tumor vasculature in a more efficient manner, resulting in an increased accumulation of those mitosomes in the interstitial spaces of tumor tissues, ${ }^{42,43}$ (3) the mitosomes as stealth liposomes exhibited prolonged half lives and sustained drug-release profiles in vivo, which could improve antitumor activity; ${ }^{44}$ and (4) the mitochondrial genes could be regulated by apoptotic inducer amlodipine, which was selectively delivered to mitochondria by antiresistant epirubicin mitosomes.

In conclusion, a new kind of cancer mitochondria-specific drug-loaded liposome was designed, antiresistant epirubicin 
mitosomes, which could provide a complete solution to the intrinsic MDR of cancers. Antiresistant epirubicin mitosomes could selectively accumulate into mitochondria of cancer cells and initiate the intrinsic pathway mediated by mitochondria through inducing the PT pore opening, resulting in release of cytochrome $\mathrm{c}$, followed by activating caspase-9, and subsequently the effector caspase-3. In vitro, antiresistant epirubicin mitosomes were effective in inhibiting the proliferation of both K562 and MDR K562/ADR cells, and in reversing drug resistance of MDR K562 cells. In vivo, antiresistant epirubicin mitosomes had the enhanced antitumor effects, and inducing apoptotic effects in the MDR K562/ADR xenografts. Therefore, antiresistant epirubicin mitosomes may provide a promising strategy for improving therapeutic efficacy in the resistant human leukemia.

\section{Authorship}

WLL contributed to the conception and design of the study. YM performed the majority of experiments and drafted the article; XXW participated in some of the experiments and revised the article. WLL revised the article and obtained the necessary funding. Other authors contributed to some of the experiments.

\section{Acknowledgments}

This work was supported by the Key Grant of Beijing Natural Science Foundation (No. 7091005), the National Natural Science Foundation of China (No. 81172991), and the National Key Science Research Program of China (973 program, 2009CB930300).

\section{Disclosure}

The authors report no potential conflicts of interest in this work.

\section{References}

1. Lautier D, Canitrot Y, Deeley RG, Cole SP. Multidrug resistance mediated by the multidrug resistance protein (MRP) gene. Biochem Pharmacol. 1996;52(7):967-977.

2. Johnstone RW, Ruefli AA, Lowe SW. Apoptosis: a link between cancer genetics and chemotherapy. Cell. 2002;108(2):153-164.

3. Gottesman MM. Mechanisms of cancer drug resistance. Annu Rev Med. 2002;53:615-627.

4. Kastrup IB, Worm J, Ralfkiaer E, Hokland P, Guldberg P, Grønbaek K. Genetic and epigenetic alterations of the reduced folate carrier in untreated diffuse large B-cell lymphoma. Eur J Haematol. 2008;80(1):61-66.

5. Mitchell P. Coupling of phosphorylation to electron and hydrogen transfer by a chemiosmotic type of mechanism. Nature. 1961;191: 144-148.

6. Murphy MP. Slip and leak in mitochondrial oxidative phosphorylation. Biochim Biophys Acta. 1989;977(2):123-141.

7. Murphy MP, Smith RA. Drug delivery to mitochondria: the key to mitochondrial medicine. Adv Drug Deliv Rev. 2000;41(2):235-250.
8. Weiss MJ, Wong JR, Ha CS, et al. Dequalinium, a topical antimicrobial agent, displays anticarcinoma activity based on selective mitochondrial accumulation. Proc Natl Acad Sci U S A. 1987;84(15): 5444-5448.

9. Brown GC, Nicholls DG, Cooper CE. Mitochondria and Cell Death. Princeton, NJ: Princeton University Press; 1999:vii-viii.

10. Hengartner MO. The biochemistry of apoptosis. Nature. 2000;407(6805): 770-776.

11. Ashkenazi A, Dixit VM. Apoptosis control by death and decoy receptors. Curr Opin Cell Biol. 1999;11(2):255-260.

12. Green DR, Reed JC. Mitochondria and apoptosis. Science. 1998; 281(5381):1309-1312.

13. Martinou JC, Desagher S, Antonsson B. Cytochrome c release from mitochondria: all or nothing. Nat Cell Biol. 2000;2(3):E41-E43.

14. Earnshaw WC, Martins LM, Kaufmann SH. Mammalian caspases: structure, activation, substrates, and functions during apoptosis. Annu Rev Biochem. 1999;68:383-424.

15. Galluzzi L, Larochette N, Zamzami N, Kroemer G. Mitochondria as therapeutic targets for cancer chemotherapy. Oncogene. 2006;25(34): 4812-4830.

16. Matsuyama S, Reed JC. Mitochondria-dependent apoptosis and cellular pH regulation. Cell Death Differ. 2000;7(12):1155-1165.

17. Green DR, Kroemer G. The pathophysiology of mitochondrial cell death. Science. 2004;305(5684):626-629.

18. Antignani A, Youle RJ. How do Bax and Bak lead to permeabilization of the outer mitochondrial membrane? Curr Opin Cell Biol. 2006;18(6): 685-689.

19. Plosker GL, Faulds D. Epirubicin: a review of its pharmacodynamic and pharmacokinetic properties, and therapeutic use in cancer chemotherapy. Drugs. 1993;45(5):788-856.

20. Li X, Ruan GR, Lu WL, et al. A novel stealth liposomal topotecan with amlodipine: apoptotic effect is associated with deletion of intracellular $\mathrm{Ca} 2+$ by amlodipine thus leading to an enhanced antitumor activity in leukemia. J Control Release. 2006;112(2):186-198.

21. Bernal SD, Lampidis TJ, McIsaac RM, Chen LB. Anticarcinoma activity in vivo of rhodamine 123 , a mitochondrial-specific dye. Science. 1983;222(4620):169-172.

22. Modica-Napolitano JS, Arille JR. Delocalized lipophilic cations selectively target the mitochondria of carcinoma cells. Adv Drug Deliv Rev. 2001;49(1-2):63-70.

23. Weissig V, Torchilin VP. Towards mitochondrial gene therapy: DQAsomes as a strategy. J Drug Target. 2001;9(1):1-13.

24. Yamada Y, Harashima H. Mitochondrial drug delivery systems for macromolecule and their therapeutic application to mitochondrial diseases. Adv Drug Deliv Rev. 2008;60(13-14):1439-1462.

25. Ying X, Wen H, Lu WL, et al. Dual-targeting daunorubicin liposomes improve the therapeutic efficacy of brain glioma in animals. $J$ Control Release. 2010;141(2):183-192.

26. Mosmann T. Rapid colorimetric assay for cellular growth and survival: application to proliferation and cytotoxicity assays. J Immunol Methods. 1983;65(1-2):55-63.

27. Liang ZH, Wu PH, Li L, Xue G, Zeng YX, Huang WL. Inhibition of tumor growth in xenografted nude mice with adenovirus-mediated endostatin gene comparison with recombinant endostatin protein. Chin Med J. 2004;117(12):1809-1814.

28. Mori M, Terui Y, Tanaka M, et al. Antitumor effect of beta2microglobulin in leukemic cell-bearing mice via apoptosis-inducing activity: activation of caspase-3 and nuclear factor-kappaB. Cancer Res. 2001;61(11):4414-4417.

29. Sun $P$, Ren XD, Zhang HW, et al. Serum from rabbit orally administered cobra venom inhibits growth of implanted hepatocellular carcinoma cells in mice. World J Gastroenterol. 2003;9(11):2441-2444.

30. Perkins C, Kim CN, Fang G, Bhalla KN. Bhalla. Arsenic induces apoptosis of multidrug-resistant myeloid leukemia cells that express BcrAbl or overexpress MDR, MRP, Bcl-2, or Bcl-xL. Blood. 2000;95(3): 1014-1022.

31. Schapira AH. Mitochondrial disease. Lancet. 2006;368:70-82. 
32. Wallace DC. The mitochondrial genome in human adaptive radiation and disease: on the road to therapeutics and performance enhancement Gene. 2005;354:169-180.

33. Szewczyk A, Wojtczak L. Mitochondria as a pharmacological target. Pharmacol Rev. 2002;54:101-127.

34. Kale AA, Torchilin VP. Environment-responsive multifunctional liposomes. Methods Mol Biol. 2010;605:213-242.

35. Weissig V, Torchilin VP. Cationic single-chain bolaamphiphiles as new materials for applications in medicine and biotechnological applications. World J Microbiol Biotechnol. 1995;11:115-131.

36. De Rosa M, Gambacorta A, Gliozi A. Structure, biosynthesis, and physicochemical properties of archaebacterial lipids. Microbiol Rev. 1986;50(1):70-80.

37. Weissig V, Lasch J, Erdos G, Meyer HW, Rowe TC, Hughes J. DQAsomes: a novel potential drug and gene delivery system made from dequalinium. Pharm Res. 1998;15(2):334-337.

38. D’Souza GG, Rammohann R, Cheng SM, Torchilin VP, Weissig V. DQAsome-mediated delivery of plasmid DNA toward itochondria in living cells. J Control Release. 2003;92(1-2):189-197.
39. Weissig V, Cheng SM, D'Souza GG. Mitochondrial pharmaceutics. Mitochondrion. 2004;3(4):229-244.

40. Twomey C, McCarthy JV. Pathways of apoptosis and importance in development. J Cell Mol Med. 2005;9(2):345-359.

41. Li P, Nijhawan D, Budihardjo I, et al. Cytochrome c and dATPdependent formation of Apaf-1/caspase-9 complex initiates an apoptotic protease cascade. Cell. 1997;91(4):479-489.

42. Dedhar S, Hannigan GE, Rak J, Kerbel RS. The extracellular environment and cancer. In: Tannock IF, Hill RP, editors. The Basic Science of Oncology. 3rd ed. New York: McGraw-Hill; 1998:198-218.

43. Gabizon AA. Selective tumor localization and improved therapeutic index of anthracyclines encapsulated in long-circulating liposomes. Cancer Res. 1992;52(4):891-896.

44. Sadzuka Y, Sugiyama I, Tsuruda T, Sonobe T. Characterization and cytotoxicity of mixed polyethyleneglycol modified liposomes containing doxorubicin. Int J Pharm. 2006;312(1-2):83-89.
International Journal of Nanomedicine

\section{Publish your work in this journal}

The International Journal of Nanomedicine is an international, peerreviewed journal focusing on the application of nanotechnology in diagnostics, therapeutics, and drug delivery systems throughout the biomedical field. This journal is indexed on PubMed Central,

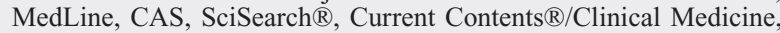

\section{Dovepress}

Journal Citation Reports/Science Edition, EMBase, Scopus and the Elsevier Bibliographic databases. The manuscript management system is completely online and includes a very quick and fair peer-review system, which is all easy to use. Visit http://www.dovepress.com/ testimonials.php to read real quotes from published authors.

Submit your manuscript here: http://www.dovepress.com/international-journal-of-nanomedicine-journal 